\title{
Sine-Gordon breathers on spatially periodic potentials
}

\author{
Angel Sánchez, ${ }^{*}$ Rainer Scharf, Alan R. Bishop, and Luis Vázquez* \\ Theoretical Division and Center for Nonlinear Studies, Los Alamos National Laboratory, Los Alamos, New Mexico 87545
}

(Received 24 October 1991)

\begin{abstract}
We have carried out an extensive simulation program to study the behavior of sine-Gordon breathers initially at rest in the presence of perturbations that are periodic in space and constant in time. We report here a number of different observed phenomena and the range of the relevant parameters (the ratio of the breather width to the perturbation wavelength and the perturbation magnitude) for which each one of them occurs. We also propose some qualitative explanations valid for certain regimes.
\end{abstract}

PACS number(s): 03.40.Kf

The study of nonlinear disordered systems has recently become the subject of a great deal of research [1]. A model that has been widely used as an approximate description of quasi-one-dimensional physical phenomena is the sine-Gordon ( $\mathrm{SG}$ ) equation (see, for instance, [2] and references therein). The effects of a large variety of perturbations on the properties of that equation have been investigated (see $[3,4]$ for recent reviews). However, starting from the seminal work by Fogel et al. [5], only a few papers have been devoted to disordered systems, corresponding to the addition of certain properly chosen inhomogeneous terms to the original equation. In particular, a simple form of inhomogeneity, which to some extent is amenable to analytical work, is a spatially periodic, external potential. The propagation of $S G$ kinks on such a potential has been previously studied by Mkrtchyan and Shmidt [6] and Malomed and Tribelsky [7], and the same problem has been analyzed as the limiting case of a spatial lattice of impurities when this lattice becomes very dense [8].

In this work we concern ourselves with the influence of these periodic potentials on the $\mathrm{SG}$ breathers. Some preliminary work has been done by Pascual [9]. We introduce this issue as an excellent example of the competition between two characteristic length scales, which is an ubiquitous phenomenon in real physical systems: In this case, these length scales are the breather width $\left(\lambda_{B}\right)$ and the perturbation period $\left(\lambda_{P}\right)$. The aim of this work is twofold. First, our immediate goal is to learn whether the particle picture of solitons, often very useful, holds or not, and, if not, when and how it breaks down due to this perturbation; second, our ultimate purpose is to apply our results to nonlinear wave propagation in potentials which are stochastic in space (this problem has been considered in [10]), viewing the periodic potential as a particular component or "color" of the noisy one. As is explained below, understanding one color is a fundamental input to treating propagation in a general color distribution. On the other hand, we are also interested in comparing the phenomena we are describing here to those caused by the same kind of potential on the nonlinear Schrödinger (NLS) equation, often viewed as a weak nonlinearity limit of the SG equation. Our investigations on this related system are planned to be reported elsewhere [11].

To approach the above issues, we have carried out a number of numerical simulations spanning large intervals of the problem parameters, namely, the lengths ratio $\left(\lambda_{B} / \lambda_{P}\right)$ and the potential strength $(\epsilon)$. We have found that there are basically three possible behaviors for the breather, largely independent of its amplitude. Thus, if $\lambda_{B}<\lambda_{P}$ (see Fig. 1) the breather. can indeed be considered a particle in the external periodic potential, and if $\lambda_{B}>\lambda_{P}$, this is still valid except that now the effective potential in which the particle moves is not the original but a renormalized one; on the contrary, if $\lambda_{B} \approx \lambda_{P}$ (competing lengths), this particlelike behavior ceases to be true even for small $\varepsilon$ values, and the breather rapidly breaks up, either into a kink-antikink $(K-\bar{K})$ pair (if its amplitude is large enough) or into two or more breathers, involving always a great amount of radiation. Interestingly, we also found that breather breakup happens also for noncompeting lengths when $\epsilon$ is above a certain threshold $\epsilon_{c}$, which depends on $\lambda_{B} / \lambda_{P}$. We have also observed for large $\lambda_{B} / \lambda_{P}$ nonradiative splitting of large-amplitude breathers into $K-\bar{K}$ pairs, which seems to have its origin in energetic considerations and not in

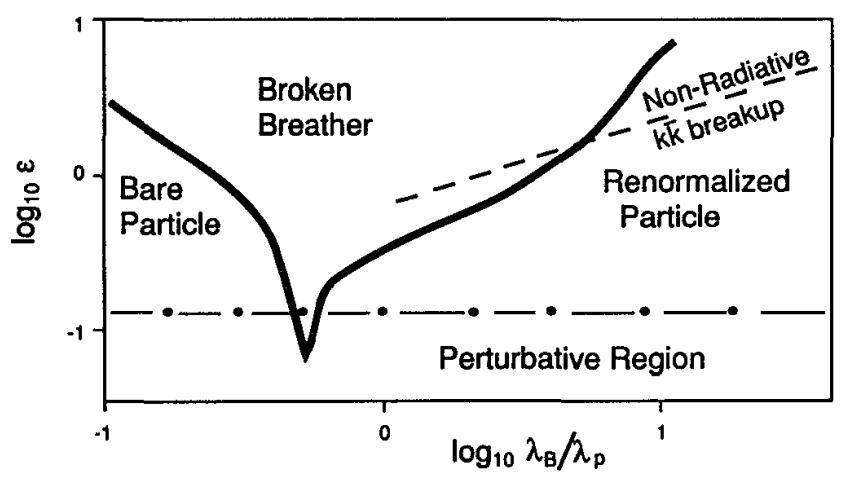

FIG. 1. Schematic "phase diagram" summarizing our main results (see text). 
the lengths competition [12]. All these phenomena are smoothed out by dynamical effects when breathers are moving, as observed in detail in the NLS model [11].

The SG equation we deal with is

$$
u_{t t}-u_{x x}+[1+\epsilon \cos (k x)] \sin u=0,
$$

and we are mainly interested in the behavior of breathers, whose expression in the unperturbed case is

$$
u^{b r}(x, t)=4 \tan ^{-1}\left(\tan \mu \frac{\sin \left[\left(t-t_{0}\right) \cos \mu\right]}{\cosh \left[\left(x-x_{0}\right) \sin \mu\right]}\right) .
$$

The length scale of the potential is given by $\lambda_{P}=2 \pi / k$. For small-amplitude breathers $(\mu \ll \pi / 2)$ the breather length scale is given by $\lambda_{B}=1 / \sin \mu$, whereas for largeamplitude breathers $(\mu \approx \pi / 2)$ one finds using Eq. (2): $\lambda_{B} \approx \operatorname{arccosh}\left\{\tan \mu \sin \left[\left(t-t_{0}\right) \cos \mu\right]\right\} / \sin \mu$.

In principle, several analytical techniques are available to tackle this problem. Nevertheless; all of them involve very difficult calculations and it turns out at last that the amount of information one can obtain from them is limited in terms of the phenomena outlined above. We will summarize them here for completeness, as well as a suggestion of further research. The first one is inverse scattering transform (IST) perturbation theory [3]. IST perturbation theory provides information in two complementary ways: the adiabatic corrections to the unperturbed breather, which neglect radiative contributions treating the breather as a particle (and leading to the same results as other, simpler collective coordinates techniques, see below), and the nonadiabatic estimations of the amount of radiation $[3,13]$. IST perturbation theory is useful in the small- $\epsilon$ regime; this restricts the interval of interest to the competing lengths region because, away from it, small- $\epsilon$ values have little effect on the breathers (see Fig. 1); it will be interesting to evaluate the radiation contribution in the competition region, which, were it large, would indicate that some instability is developed by the breather when its length is similar to the perturbation wavelength. This is quite complicated, but there is progress in that direction in the case of the NLS equation with spatially periodic potentials [11].

A different procedure, which has the advantage of its easy physical interpretation, is the already mentioned one of the collective coordinates (see [14] for a very recent account on these techniques). We will show that they indeed provide some useful information, without overly involved algebra. With the basic assumption that the effect of the potential on the breather is to allow it to move, without changing its shape, it is possible to derive [further detail is planned to be given elsewhere [12], but the basis is the fact that the evolution under Eq. (1) conserves energy, see, e.g.; [3]] the following effective potential for the breather coordinate at rest:

$$
\begin{aligned}
V_{\mathrm{eff}}\left(x_{0}, z\right)= & \frac{4 \pi \sinh z \cos \left(k x_{0}\right)}{\sin \mu \cosh ^{2} z \sinh (K \pi / 2)} \\
& \times\left(\frac{\sin (K z)}{\cosh z}+K \cos (K z) \sinh z\right),
\end{aligned}
$$

where $K \equiv k / \sin \mu=\lambda_{B} / \lambda_{P}$ and $z$, defined by $\sinh z \equiv$ $\tan \mu \sin (t \cos \mu)\left(t_{0}=0\right)$, is a measure for the distance between the kink and the antikink bound in the breather. From this expression, it immediately turns out that if the collective coordinate approach is valid, for small $\lambda_{B} / \lambda_{P}$ the breather will move in an effective potential which is similar to, and of the order of magnitude of, the one entering Eq. (1), namely,

$$
\begin{aligned}
V_{\mathrm{eff}}\left(x_{0}, z\right)= & \frac{8 \sinh z \cos \left(k x_{0}\right)}{\sin \mu \cosh ^{2} z} \\
& \times\left(\frac{\sin (K z)}{K \cosh z}+\cos (K z) \sinh z\right),
\end{aligned}
$$

whereas if $\lambda_{B} / \lambda_{P}$ becomes very large, the resulting effective potential $V_{\text {eff }}\left(x_{0}, z\right) \propto \cos \left(k x_{0}\right) / \sinh (K \pi / 2)$ is negligible for practical purposes. The question as to whether there is a regime in which such an effective potential makes physical sense can only become clear after comparing to the simulations. However, some agreement can be expected for small $\epsilon$, at least when $\lambda_{B} / \lambda_{P}$ is far away from 1 (i.e., the lower left regions of Fig. 1). Unfortunately, Eq. (3) also points out some of the main obstacles to any analytical treatment, namely, the breather-time dependence; obtaining a similar effective potential but for a moving breather is a harder task.

There is still another way to obtain insight, but it has the drawback that it only applies to small-amplitude breathers, while the two summarized above do not impose a priori any restriction on the breather amplitude. This approach is based on the well-known fact that a perturbed NLS equation can be derived from Eq. (1) to describe breathers in the limit of large frequency (equivalently small amplitude) $\omega_{B}=\cos \mu \lesssim 1$. To achieve this, it is enough to look for solutions of $\mathrm{Eq}$. (1) of the form $u(x, t)=\delta A(X, T) e^{i t}+$ c.c. (c.c. stands for complex conjugate) where $\delta$ is some small parameter, and $X=\delta x$, $T=\delta^{2} t$; substitution of this Ansatz in Eq. (1) and the change $A \rightarrow 2 \psi, r \rightarrow-2 T$ yield, to order $\delta^{3}$,

$$
i \psi_{\tau}+\psi_{X X}+2|\psi|^{2} \psi=\psi \sin \left(\frac{k}{\epsilon} X\right)
$$

At a step in this procedure, one has to specify how $\delta$ and $\epsilon$ relate to each other. To reach Eq. (5), this must be set to $\epsilon \approx \delta^{2}$, which means that Eq. (5) is only valid for small-amplitude breathers on even smaller perturbations. This limitation is often overlooked, and we believe that keeping it in mind is crucial to make a correct comparison of NLS solitons to small-amplitude SG breathers. It is possible to remove this restriction by starting from more sophisticated Ansätze, including terms in $\delta^{2}$ and $\delta^{3}$ (see [15]; see also [16]); this technique leads to a more complicated perturbed NLS equation, given by

$$
i \psi_{r}+\psi_{X X}+2|\psi|^{2} \psi=\frac{2}{k^{2}} \psi \sin ^{2}\left(\frac{k}{\epsilon} X\right)+\frac{4}{k^{2}} \psi_{X} \cos \left(\frac{k}{\epsilon} X\right)
$$

which, in turn, only requires $\epsilon \approx \delta$ for it to be valid up to $O\left(\delta^{3}\right)$. We see that the comparison of SG and NLS results is not at all trivial, and seriously depends on the 
perturbation regime one is interested in. On the other hand, both Eqs. (5) and (6) imply two interesting consequences: first, there can be an "effective renormalization" of the wave number of the perturbation as a function of its strength, and, second, the effects of it on the breather will show the same wave number as the original periodic potential (in the original $x$ coordinate). Equation (6) gives the additional information that the gradients of the wave field are going to play an important role in the evolution of the breather. A more detailed analysis of both equations can provide some hints of mechanisms that can be acting in larger-amplitude breathers, and will be addressed elsewhere.

Let us now move to the main topic of this report, namely, the numerical simulations we have carried out on Eq. (1). Our numerical procedure was based on the Strauss-Vázquez scheme [17], which recently has turned out to be very appropriate to deal with perturbed nonlinear Klein-Gordon equations (see [18] and references
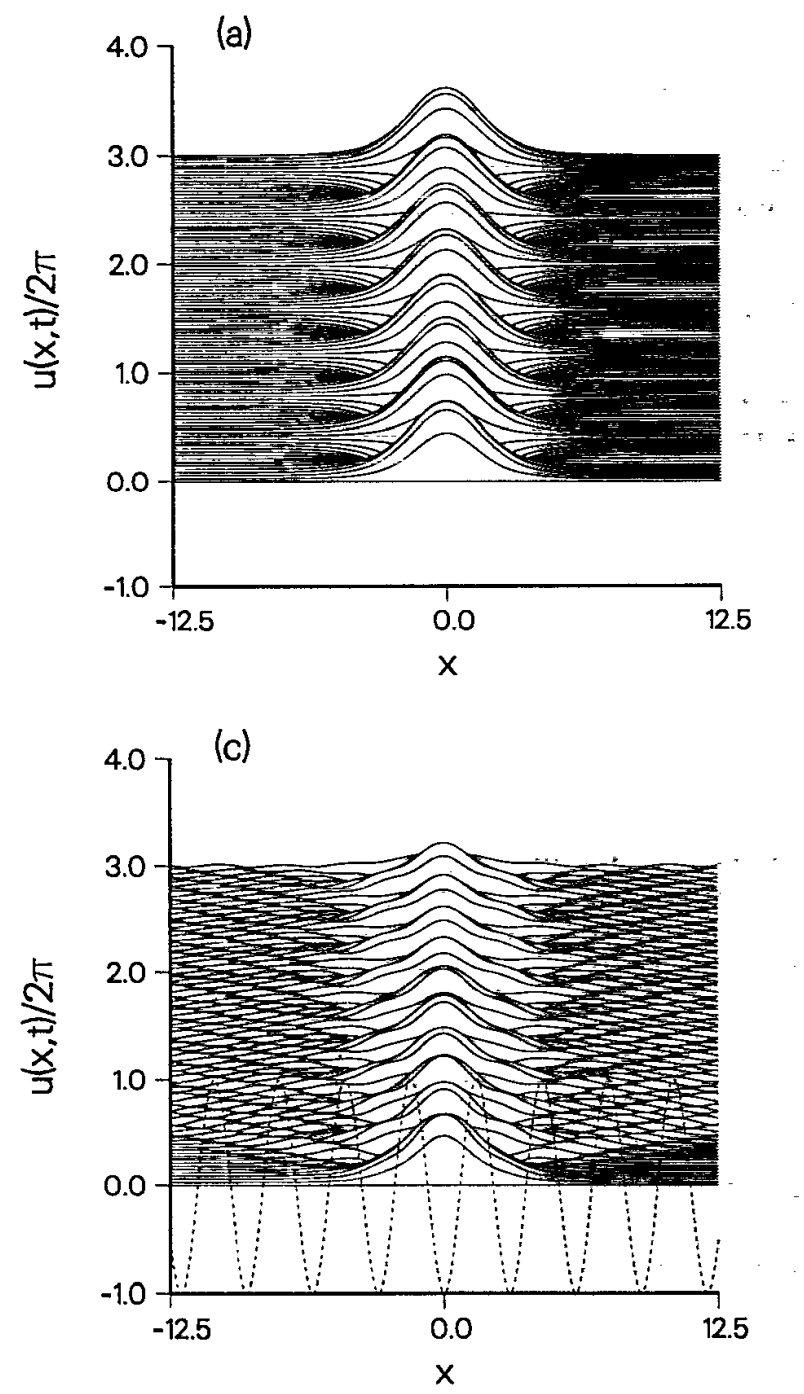

therein). As we are interested in the effect of the perturbation on breathers, we start from an unperturbed one as the initial condition; in most of the simulations the breather is placed at $x=0$ (a maximum of the periodic potential) but some other locations (bottom, inflection points) have been also considered. Notice that the problem can be different if the breather is adiabatically (i.e., with $\epsilon$ increased slowly from zero) placed on the potential. For the current report, we restrict ourselves to breathers initially at rest, in an attempt to understand the main features of their evolution. This is a prelude to the dynamical problem, which, as we already stated, is expected to exhibit some smoothing of the static breather's instabilities. The boundary conditions are taken to be periodic. The spatial mesh size is chosen so that the equations are in the continuum regime, without any discrete effect. The breather evolution is followed to long times, usually of the order of $t=500$ in our dimensionless units which amount to approximately
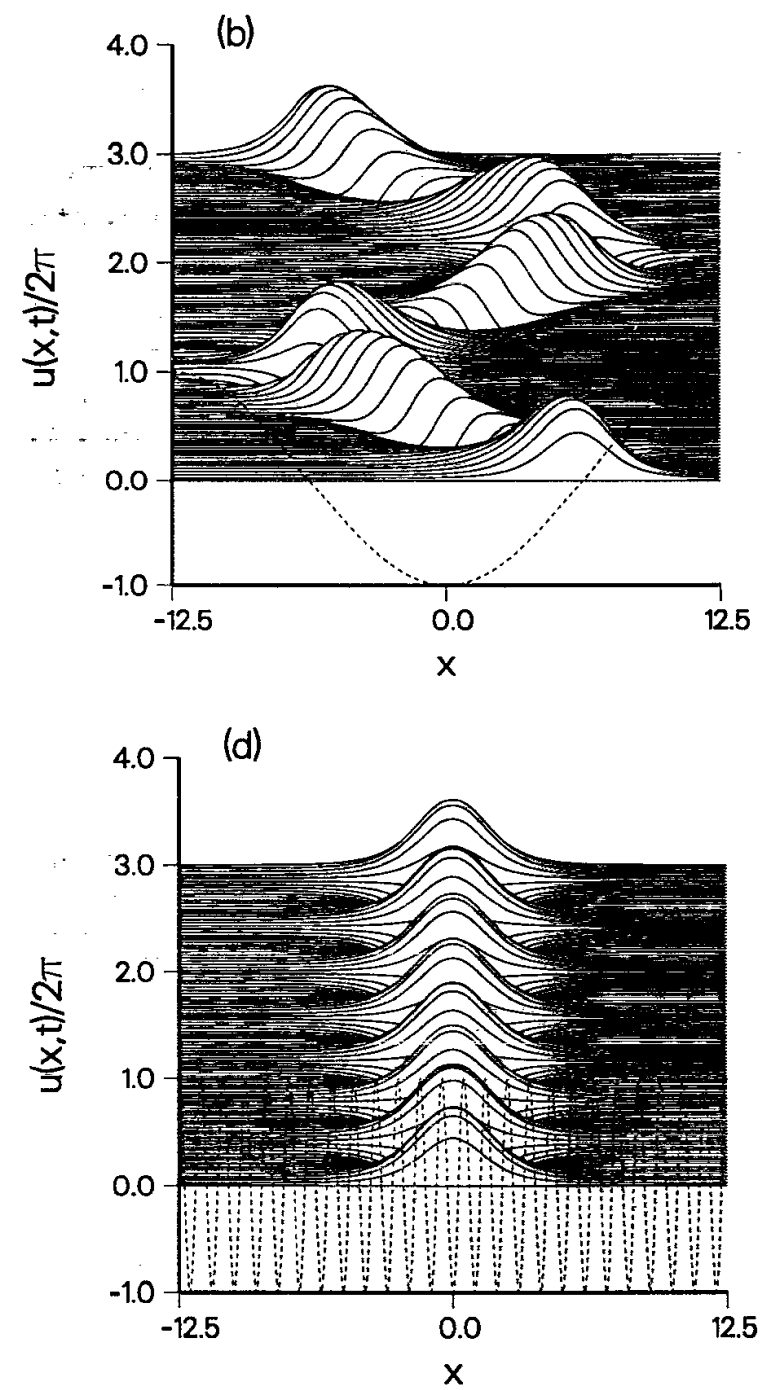

FIG. 2. Numerical simulations of breathers for the SG Eq. (1). Shown is $u(x, t)$, the shape of the breather with the inital condition given by Eq. (2). Integration time $0 \leq t \leq 100$, time step $\Delta t=0.025$, spatial step $\Delta x=0.05$. Breather parameters: $\mu=1, t_{0}=0$. (a) No potential: $\epsilon=0, x_{0}=0$. (b) Long-wavelength potential: $\epsilon=-0.5, k=2 \pi / 25, x_{0}=6$. (c) Breather breakup for competing length scales: $\epsilon=-0.5, k=2 \pi / 3, x_{0}=0$. (d) Short-wavelength potential: $\epsilon=-0.5, k=2 \pi, x_{0}=0$. 
25000 time steps.

In Fig. 2 we show some typical results of our numerical simulations, from a breather behaving in a particlelike manner $\left(\lambda_{B} \ll \lambda_{P}\right)$, over a breather decaying into radiation due to competition of length scales $\left(\lambda_{B} \approx \lambda_{P}\right)$, to a breather smoothing over a short-wavelength potential $\left(\lambda_{B} \gg \lambda_{P}\right)$. In Fig. 3 we show a breather breaking up into kink and antikink upon a small change of $\mu$.

The outcome of our numerical simulation program is summarized in Fig. 4, which completes the qualitative picture we already presented in Fig. 1 . We start by describing the small- $\epsilon$ regime, equivalently, the lower part of the "phase diagram" of Fig. 4, for breathers starting at a maximum of the potential, $x=0$. We have found that when the initial breather is far from the value $\lambda_{B} / \lambda_{P}=1$, it remains unbroken. By this we mean that it is altered
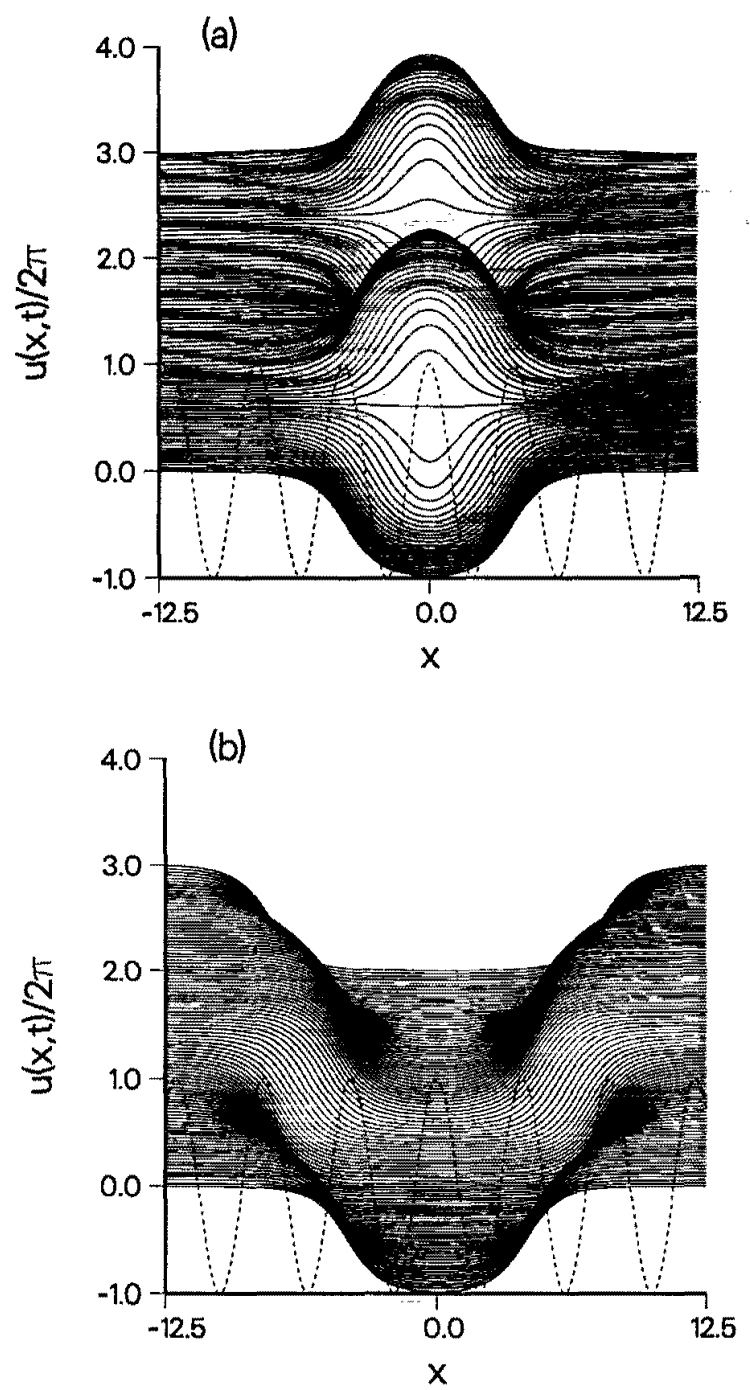

FIG. 3. Numerical simulations of breathers for the SG Eq. (1). Shown is $u(x, t)$, the shape of the breather. Integration time $0 \leq t \leq 100$, time step $\Delta t=0.025$, spatial step $\Delta x=$ 0.05 . Potential parameters: $\epsilon=0.1, k=\pi / 2$. Breather parameters: $x_{0}=0, t_{0}=\pi / 2 \cos \mu$. (a) Distorted breather: $\mu=1.53$. (b) Breakup into kink and antikink: $\mu=1.54$.
- with respect to the initial breather but still it keeps its coherence and behaves like a single elementary excitation. The main changes it undergoes are a modulation of its shape, which develops "ripples" with the same period of the perturbing potential, and an increase of the breathing frequency. It is also worth noticing that it emits a certain amount of radiation at the very beginning of the evolution, but after this transient and achieving a suitable shape, radiation ceases completely. Far from $\lambda_{B} / \lambda_{P}=1$ we have checked that there is a good qualitative agreement with the predictions of the effective potential in Eq. (3): Indeed, when $\lambda_{B} / \lambda_{P} \rightarrow 0$, the breather behaves very much like a particle, including features such as being eventually removed from its metastable initial state by numerical roundoff errors and then sliding down to one of the two neighboring wells; on the other hand, when $\lambda_{B} / \lambda_{P}$ is large, the effects on the breather both on its shape, its frequency, and its center position become barely visible. We also note that for these small $\epsilon$ values, the shape of small-amplitude breathers is very similar to the predictions arising from Eq. (6) of a modulated envelope $\psi(X, \tau)$. With respect to the initial position, moving it to a bottom of the periodic potential results in the same basic behavior, whereas any other starting point will give rise to breathers showing local differences in breathing frequency (when $\lambda_{B} / \lambda_{P}$ is small, otherwise again the potential influence is negligible) in addition to the above-described, mainly particlelike characteristics.

This picture changes dramatically as the initial breather approaches the competing lengths region $\lambda_{B} / \lambda_{P} \approx 1$. In this regime, the breather is no longer stable: The radiating transient ends up in a huge amount of radiated energy and in a rapid decay of the breather or its breakup either into two or more breathers or into a $K-\bar{K}$ pair. Sometimes this breakup is even more spectacular: The initial breather jumps over several potential barriers and finishes breaking into several breatherlike excitations, hardly distinguishable from radiation. The whole process seems to happen in a time of the order of magnitude of 10 or less in dimensionless units, and it is important to stress that even rather small values of $\epsilon$ (around 0.1) can give rise to this clearly nonperturbative behavior. A remark is in order concerning the breaking into $K-\bar{K}$, namely, that this decay channel seems to be available only for large-amplitude breathers $(\mu \approx 1.5$ the ones we studied, where the separatrix from $K-\bar{K}$ for the unperturbed system is $\mu=\pi / 2$ ). In connection with this, another important result we have obtained in this $\lambda_{B} / \lambda_{P} \approx 1$ region is the extreme sensitivity of the outcome on the precise- $\epsilon$ value: Small changes in this parameter can lead, for large-amplitude breathers, to breaking into breathers, into $K-\bar{K}$, or into radia tion [see, e.g., the sequence at $\log _{10}\left(\lambda_{B} / \lambda_{P}\right)=0.5$ in Fig. 4]; analogously, this dependence appears for loweramplitude breathers in that they can decay to breathers or just radiation. Finally, typically this radiated energy is eventually trapped in the wells of the potential, forming some small-amplitude, localized excitations.

Having summarized our main findings for small $\epsilon$, we now describe the full "phase diagram" in Fig. 4. Briefly, the same breakup phenomena arise for each value of 


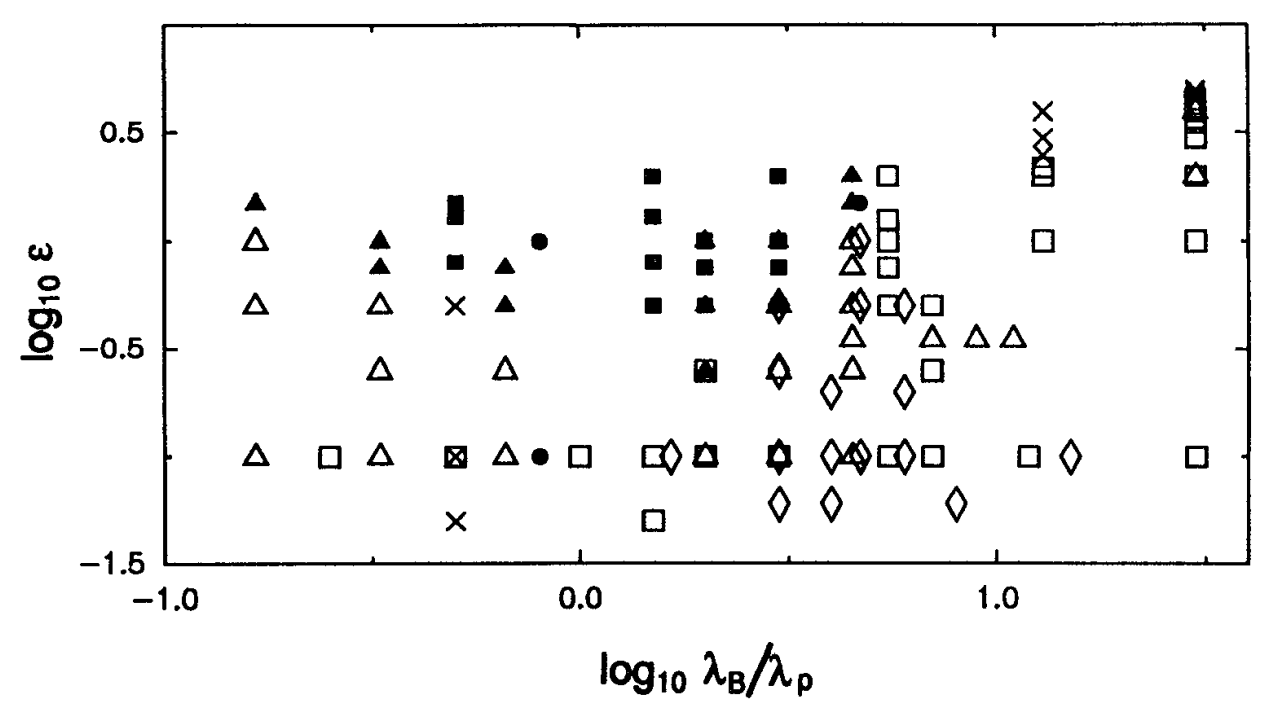

FIG. 4. The "phase diagram" as obtained from numerical simulations (cf. Fig. 1). Squares are results for large-amplitude breathers $(\mu=1.5)$, triangles for intermediate-amplitude breathers $(\mu=0.5)$, and diamonds and circles for small-amplitude breathers $(\mu=0.1)$. Empty symbols mean that the breather remained unbroken, though distorted, whereas full symbols indicate breaking up. Crosses stand for breather breakup into $K-\bar{K}$ (see text).

$\lambda_{B} / \lambda_{P}$ for $\epsilon>\epsilon_{c}\left(\lambda_{B} / \lambda_{P}\right)$; the dependence of $\epsilon_{c}$ on $\lambda_{B} / \lambda_{P}$ can be approximately seen from Fig. 4 as the boundary between black and white points. The value $\epsilon_{c}$ grows as we move further from the critical competing lengths region $\lambda_{B} / \lambda_{P} \approx 1$, and, for small or large $\lambda_{B} / \lambda_{P}$ values, we have to go to values of rather large $\epsilon$, even larger than 1 and thus allowing for the coefficient of $\sin u$ in Eq. (1) becoming negative (in other words, the natural frequency of the system can be imaginary). This is what we already depicted in the schematic Fig. 1. However, the real situation appearing from the simulations is more complicated, because there is not a single critical value but, rather, a narrow region or sequence of $\epsilon$ values for each $\lambda_{B} / \lambda_{P}$ below which breathers are preserved, above which breathers are broken, and inside which the precise outcome (breaking or nonbreaking) strongly depends on the chosen $\epsilon$. Furthermore, large-amplitude breathers undergo a different process: They can nonradiatively break up into $K-\bar{K}$ pairs, nonradiatively meaning that the process is smooth and that we have not seen an appreciable quantity of radiation in the system (see Fig. 3). This process is not necessarily correlated with the competition of lengths, because the resulting $K-\bar{K}$ pair is made up of kinks, each one of which may cover several wavelengths of the perturbation potential, aside from the lack of radiation characteristic of the competing lengths mechanism. This region can be seen in Fig. 4 as the crosses near the rightmost edge of the $\lambda_{B} / \lambda_{P}$ axis.

We can now try to give a global picture of the result of our study of Eq. (1). We believe that our "phase diagram" (Figs. 1 and 4) indeed describes the parameters regimes in which the system scales to a situation where either nonlinearity is dominant (below the solid line in Fig. 1, white zone in Fig. 4) or disorder, in the sense of inhomogeneity, is dominant (above the solid line in
Fig. 1, black zone in Fig. 4). Besides, we can conclude that this periodic potential is actually very relevant to the study of general disorder, i.e., most random (excluding probably extreme cases like white noises) potentials, because it shows that for small perturbations the only important length scale of such a noisy medium would be the one of the length of the excitation that one is trying to propagate (in our case, the breather length), whereas all other "colors" in the stochastic perturbation are either smoothed out or have only adiabatic effects. They have very little influence on nonlinear excitations, aside, of course, from the long-wavelength Fourier components which will drive the system as a whole in a particle sense.

The underlying mechanism responsible for this length selection is not clear at present. From our work we have learned that it is by no means a global one, e.g., the energy injected in the system by just placing the breather in the potential. These global quantities are in most parameter regions very small and irrelevant. It is likely that local equilibria between the extra potential energy and the wave-field gradients are the main drivers of the breather breakup. This interpretation arises both from the simulations, that show how these gradients grow as $\epsilon$ increases, and from the approach given by Eq. (6) which shows that $\psi_{X}$ is playing a role in the smallamplitude breather evolution. On the contrary, global energetic considerations are more suitable explanations of the nonradiative, $K-\bar{K}$ breakup of large-amplitude breathers, as we will report elsewhere [12]. We also want to stress again the fact that we have performed similar simulations on the NLS system [11] and find the same kind of "phase diagram," which is a good indication that NLS-like approaches to the SG breather problem can be helpful: for instance, Eqs. (5) and (6) show how the potential strength can enter through a renormalization of 
the wave number. This is in accordance with the breakup phenomena on the $\lambda_{B} / \lambda_{P}>1$ side of the abscissa of our "phase diagram": strong potentials of large wave number are effectively leading to behaviors like those of smaller wave-number perturbations, thus causing breakup processes to be activated. On the other side of the axis $\left(\lambda_{B} / \lambda_{P}<1\right)$ such an $\epsilon$ renormalization would lead to spatial intervals of almost constant potential, which, provided they were large and extended enough, would prevent breathers from existing inside them, this being not a length competition but a local change in the allowed breather frequencies.

Although Eq. (1) is rather difficult to study analytically, we hope to obtain further insight into the competing lengths mechanism along the following lines: The NLS-like equations (5) and mostly (6) look like promising points from which to obtain information on the radiative mechanism governing small-amplitude breather breakup. We have shown enough evidence that this mechanism is quite general, with the only possible exception of largeamplitude breathers where a $K-\bar{K}$ decay channel is available. Perturbation theory for these equations would then tell us something about the small- $\epsilon$ region of the "phase diagram." The limiting lines between breaking and nonbreaking for large $\epsilon$ (equivalently for $\lambda_{B} / \lambda_{P}$ far away from 1) might be understood through numerical linear stability analysis of the perturbed breather shape, studying how the corresponding spectrum evolves with $\epsilon$; the main problem of such an approach is the time dependence of the breather, but either a suitably chosen averaging or a good Ansatz for its shape may remove this difficulty. Lastly, a complementary view would be to simulate the same problem, Eq. (1), but starting from plane waves of different wavelengths instead of breathers; such a numerical work would give a different perspective of the length competition. This length competition will be more relevant for certain experimental situations (i.e., more uniform energy input) and will address the formation of solitons from modulational instabilities in the presence of disorder. We envisage scaling to linear disorder fixed points (localization) for strong disorder but to nonlinear fixed points (soliton formation) for sufficiently strong nonlinearity and length competition. If solitons are formed, the scenario discussed earlier in this report takes over.

In conclusion, we have completed an exploratory program of numerical work on SG breathers initially at rest on spatially periodic potentials. We have found that low-amplitude perturbations are only relevant when their length scale is of the order of that of the breather, leading to its breakup. For other length ratios perturbation strengths above a zone of critical values are needed to give rise to the same effects. This behavior does not depend on the breather amplitude except when this amplitude opens the $K-\bar{K}$ breakup channel. Small-amplitude SG breathers and NLS solitons are seen to behave quite similarly. In this sense the behavior of breather solitons in mono-color spatial disorder is a key component to understanding their transport in disorder of general color. This preliminary work allows us to propose some explanations for this group of phenomena which are qualitatively correct, and to select further research lines capable to complete and make our picture quantitative. Work along these lines is in progress $[11,12]$.

A.S. and L.V. acknowledge partial financial support from the Dirección General de Investigación Científica. y Técnica (D.G.I.C.yT.) of Spain, through Project No. MAT90-0544, as well as the warm hospitality of the Los Alamos National Laboratory. We enjoyed fruitful discussions with Yuri Kivshar and Pedro J. Pascual. This work has been performed under the auspices of the U.S. Department of Energy.
* Permanent address: Departamento de Física Teórica I, Facultad de Ciencias Físicas, Universidad Complutense, E-28040 Madrid, Spain.

[1] Disorder and Nonlincarity, edited by A. R. Bishop, D. K. Campbell, and St. Pnevmatikos, Springer Proceedings in Physics Vol. 39 (Springer-Verlag, Berlin, 1989); Nonlinearity with Disorder, edited by F. Kh. Abdullaev, A. R. Bishop, and St. Pnevmatikos, Springer Proceedings in Physics (Springer-Verlag, Berlin, in press).

[2] A. R. Bishop, J. A. Krumhansl, and S. E. Trullinger, Physica 1D, 1 (1980).

[3] Yu. S. Kivshar and B. A. Malomed, Rev. Mod. Phys. 61, 763 (1989).

[4] A. Sánchez and L. Vázquez, Int. J. Mod. Phys. B 5, 2825 (1991).

[5] M. B. Fogel, S. E. Trullinger, A. R. Bishop, and J. A. Krumhansl, Phys. Rev. Lett. 36, 1411 (1976); Phys. Rev. B 15, 1578 (1977); J. F. Currie, S. E. Trullinger, A. R. Bishop, and J. A. Krumhansl, ibid. 15, 5567 (1977).

[6] G. S. Mkrtchyan and V. V. Shmidt, Solid State Commun.
30, 791 (1979).

[7] B. A. Malomed, in Solitons and Applications, edited by V. G. Makhankov, V. K. Fedyanin, and O. K. Pashaev (World Scientific, Singapore, 1990); B. A. Malomed and M. I. Tribelsky, Phys. Rev. B 41, 11271 (1990).

[8] Yu. S. Kivshar and B. A. Malomed, Phys. Lett. 111A, 427 (1985).

[9] P. J. Pascual (unpublished).

[10] P. J. Pascual, L. Vázquez, St. Pnevmatikos, and A. R. Bishop, in Disorder and Nonlinearity (Ref. [1]); A. Sánchez, Ph. D. thesis, Universidad Complutense, 1991.

[11] R. Scharf and A. R. Bishop (unpublished).

[12] R. Scharf, Yu. S. Kivshar, A. Sánchez, and A. R. Bishop, Phys. Rev. A 45, R5369 (1992).

[13] Yu. S. Kivshar, A. M. Kosevich, and O. A. Chubykalo, Phys. Lett. A125, 35 (1987).

[14] R. Boesch and C. Willis, Phys. Rep. (to be published).

[15] S. R. Phillpot, A. R. Bishop, and B. Horovitz, Phys. Rev. B 40, 1839 (1989).

[16] T. Kawahara, J. Phys. Soc. Jpn. 35, 1537 (1973); A. M. 
Kosevich and A. S. Kovalev, Zh. Eksp. Teor. Fiz. 67, 1793 (1974) [Sov. Phys. JETP 40, 891 (1975)]; R. Dashen, B. Hasslacher, and A. Neveu, Phys. Rev. D 11, 3424 (1975).

[17] W. Strauss and L. Vázquez, J. Comp. Phys. 28, 271
(1978).

[18] A. Sánchez, L. Vázquez, and V. V. Konotop, Phys. Rev. A 44, 1086 (1991). 